\title{
The Prognosis of Late Presenters in the Era of Highly Active Antiretroviral Therapy in Serbia
}

\author{
Djordje Jevtović $^{1}$, Dubravka Salemović ${ }^{1}$, Jovan Ranin ${ }^{1}$, Branko Brmbolić ${ }^{1}$, Ivana Pešić-Pavlović ${ }^{1}$, \\ Sonja Žerjav, ${ }^{1}$ and Olgica Djurković-Djaković ${ }^{*}, 2$
}

\author{
${ }^{1}$ Institute for Infectious \& Tropical Diseases, University of Belgrade School of Medicine, Belgrade, Serbia \\ ${ }^{2}$ Institute for Medical Research, University of Belgrade, Belgrade, Serbia
}

\begin{abstract}
To examine the prognosis of patients who present with very advanced HIV-induced immunodeficiency, and their response to highly active antiretroviral therapy (HAART), a series of 101 treatment naïve patients from the Serbian cohort of HIV infected patients, who presented with a CD4 count of $\leq 50 / \mu \mathrm{L}$ before commencing HAART, was retrospectively analyzed and factors influencing response to HAART and survival investigated. After a mean of three years (range 1-9) of treatment with PI-based and/or NNRTI-based regimens, a favorable response was achieved in 54.5\% of the patients, treatment failure occurred in $13.9 \%$, while $31.7 \%$ had a dissociative immunological/virological response. The overall estimated survival was eight years. Achievement of undetectable viremia during treatment appeared life saving $(\mathrm{OR}=42.5,95 \% \mathrm{CI} 7.1-251.9, P=0.000$, as was a rise in CD4 cell count to over $200 / \mu \mathrm{L}(\mathrm{OR}=6.4,95 \% \mathrm{CI} 1.2$ $31.8, P=0.023)$. However, undetectable viremia was the single predictor of longer survival $(\mathrm{OR}=42.5,95 \% \mathrm{CI} 7.1-$ 251.9, $P=0.000$ ), regardless of the level of immune reconstitution (log rank, $P=0.31$ ). Late presenters had a high probability of developing the metabolic syndrome while on HAART, with a median time to hyperlipidemia and lypodystrophy of 5 and 6 years, respectively. We conclude that late presenters on HAART may have a good prognosis, a prerequisite for which is sustained undetectable viremia regardless of the immune recovery.
\end{abstract}

Keywords: Late presenters, HAART, survival.

\section{INTRODUCTION}

A major advance in HIV disease treatment occurred with the introduction of highly active antiretroviral treatment (HAART) in 1996. HAART regimens comprise two nucleoside reverse transcriptase inhibitors (NRTIs) and a HIV protease inhibitor (PI) or non-nucleoside reverse transcriptase inhibitor (NNRTI), potent enough for complete and prolonged cessation of viral replication to allow reconstitution of the immune system [1-3]. Although both NRTIs and NNRTIs inhibit reverse transcriptase, their action through different mechanisms (NRTIs are phosphorylated to active metabolites and compete for incorporation into viral DNA, terminating synthesis of DNA chains, while NNRTIs bind directly to the reverse transcriptase enzyme active site) provides an enhanced effect. Immune recovery has even been achievable in severely ill patients. An overall reduction in late stage complications of HIV infection was associated with an impressive improvement in patient survival and quality of life [4-6]. However, we still do witness late presenters i.e. patients diagnosed with HIV infection in the advanced stage of HIV-related immunodeficiency, when they often suffer life-threatening opportunistic infections and/or tumors [7-12]. Most pertinent research investigated reasons for late presentation, while some also addressed the prognosis of late presenters on HAART [5-16].

*Address correspondence to this author at the Institute for Medical Research, University of Belgrade, Dr. Subotića 4, P.O. Box 102, 11129 Belgrade, Serbia; Tel: +381112685 788; Fax: +381 112643 691;

E-mail: olgicadj@imi.bg.ac.rs
Serbia is a country of low AIDS prevalence, with, however, a low HIV-testing rate. This is why a number of patients present late in the course of HIV disease. Indeed, between January 1998 and December 2007, late presenters have accounted for $17.1 \%$ of patients referred to the national HIV/AIDS Centre. We conducted a retrospective analysis of late presenters in whom HAART was initiated to study the factors influencing response to treatment and survival.

\section{METHODS}

\section{Patients}

The study series involved 101 treatment naïve patients from the Serbian cohort of HIV infected patients treated at the HIV/AIDS Centre at the Institute for Infectious and Tropical Diseases in Belgrade, who presented between January 1998 and December 2007 with most advanced HIVinduced immunodeficiency. Inclusion criteria consisted of adult HIV infection (stages A-C according to the 1993 Centers for Disease Control case definition criteria) [17], CD4 cell count below $50 / \mu \mathrm{L}$ ("late presenters"), and initiation on HAART after diagnosis. Follow-up was continued until the last visit, death or 31 December 2007. Patients who did not survive the index AIDS defining condition were excluded from the study. Consent for participation was obtained from all, and the study was approved by the Clinical Centre of Serbia Ethics Committee.

Demographic characteristics, AIDS diagnosis prior to HAART initiation, co-infection with hepatitis $\mathrm{C}$ virus (HCV), along with parameters associated with response to HAART including plasma viral load and CD4+ cell count as 
well as the duration of treatment, were all assessed as variables with possible influence on treatment efficacy and survival. Influence of antiretroviral drug classes used as HAART components was also analyzed.

\section{Clinical and Laboratory Data}

The immunological and virological responses to HAART were evaluated by measuring plasma viral load $(\mathrm{pVL})$ and $\mathrm{CD} 4+\mathrm{T}$ cell counts. Criteria for the type of response to HAART were as follows: the response was considered favorable in case of achievement of a sustained $\mathrm{pVL}$ reduction to undetectable values, along with $\mathrm{CD} 4$ cell count increases to above $200 / \mu \mathrm{L}$. In contrast, treatment failure was defined as a pVL over $2.9 \log _{10}$ copies of HIV RNA/ml of plasma regardless of immunological improvement. In addition to these two clear-cut types of response to HAART, dissociation between immunological and virological responses to HAART occurred at times, defined as achievement of undetectable viremia during treatment but without a rise in CD4 cell counts to above $200 / \mu \mathrm{L}[18,19]$. The threshold of $200 \mathrm{CD} 4$ cells $/ \mu \mathrm{L}$ was taken as one decreasing the risk of serious opportunistic infections. However, the results were additionnally analyzed according to a threshold of CD4 $>400$ cells $/ \mu \mathrm{L}$, which is one approaching normal values, and has been used in previous analyses of our cohort [19].

\section{Laboratory Methods}

CD4 cell counts and viral loads were measured in peripheral blood samples drawn at presentation and every 46 months during follow-up. CD4 cells were quantified by flow cytometry. Plasma HIV-1 RNA loads were measured by a quantitative reverse transcriptase polymerase chain reaction (Roche Molecular Systems, Branchburg, NY, USA), which has a lower limit of detection of 50 copies $/ \mathrm{mL}(1.7$ $\log _{10}$ ).

\section{Statistics}

All analyses were performed using an electronic database organized in the SPSS (version 11.5) statistical package. The association between the type of response to HAART, and variables including baseline and end-point CD4 cell counts and $\mathrm{pVL}$, type of HAART regimen, and age above 40, were assessed using the chi-square test and univariate and multivariate logistic regression. Odds ratios (ORs) and the corresponding $95 \%$ confidence intervals (CIs) were used as estimators of relative risk for HAART failure, and/or lethal outcome. The Kaplan-Meier product limit method was used to determine the probability of reaching immunological and virological improvement, and survival, as well as to assess the probability of developing drug toxicities over time. Oneway analysis of variance (ANOVA) was used to compare means. The level of statistical significance was 0.05 .

\section{RESULTS}

\section{Patient and Treatment Characteristics}

Baseline characteristics of all patients are presented in Table 1.

Late presenters were more likely to be male $(72.3 \%)$. Regarding transmission risk factors, the frequency of men having sex with men (MSM), heterosexuals and IV drug users was similar. There was only one patient who got infected vertically.

Table 1. Baseline Characteristics of a Series of 101 Late Presenters

\begin{tabular}{|l|l|}
\hline Variable & $\mathbf{N}(\mathbf{\%})$ \\
\hline Female & $28(27.7)$ \\
\hline Male & $73(72.3)$ \\
\hline Age & $44 \pm 9.8$ \\
\hline Risk Group & \\
MSM & $43(42.6)$ \\
heterosexuals & $28(27.7)$ \\
IVDU & $29(28.7)$ \\
vertical transmission & 1 \\
\hline Age over 40 & $57(56.4)$ \\
\hline Clinical AIDS & $71(70.3)$ \\
AIDS Defining Condition & \\
TB & $23(32.4)$ \\
PCP & $15(21.1)$ \\
CE & $10(14.1)$ \\
WS & $9(12.7)$ \\
CNM & $4(5.6)$ \\
TGE & $4(5.6)$ \\
CMV & $3(4.2)$ \\
ADC & $2(2.8)$ \\
KS & $1(1.4)$ \\
\hline HCV co-infection & $26.3 \pm 18.0$ \\
\hline
\end{tabular}

Men having sex with men (MSM); Intravenous drug users (IVDU); TB (Tuberculosis); PCP (P. carinii pneumonia); CE (Candida esophagitis); WS (Wasting syndrome); CNM (C. neoformans meningitis); TGE (Toxoplasma gondii encephalitis); CMV (Cytomegalovirus disease); ADC (AIDS dementia complex); KS (Kaposi sarcoma)

Treatment characteristics are presented in Table 2. The overall duration of HAART was $2.86 \pm 1.80$ years (range 19 ), but varied according to regimen. HAART regimens included combinations of two NRTIs as a treatment backbone, with (I) one or two PI (taken by $34.6 \%$ of all patients), (II) one NNRTI (35.6\%), and (III) combinations of drugs from all three classes or triple NRTI regimens during some period of their treatment $(29.7 \%$ switched multiple regimens during follow-up). Regimen sequencing was performed in case of virological and/or immunological failure, and also in some instances to simplify treatment by lowering pill burden. Antiretrovirals available in Serbia included zidovudine (AZT), lamivudine (3TC), didanosine (ddI), stavudine (d4T), abacavir (ABV), nevirapine, efavirenz, indinavir, saquinavir, nelfinavir, lopinavir/r, ritonavir(r), and enfuvirtide. Ritonavir was used for PI boosting only. Genotypic resistance testing was not routinely performed; thus, subsequent regimens were composed on the basis of standards of care [20]. However, three extensively pre-treated patients, after being diagnosed with multi drugclasses' resistant virus, were switched to newer drugs, such as enfuvirtide, darunavir/ $\mathrm{r}$, and atazanavir/ $\mathrm{r}$ (the latter two as a compassionate treatment). 
Table 2. Characteristics of HAART in a Series of 101 Late Presenters

\begin{tabular}{|l|c|c|}
\hline \multicolumn{1}{|c|}{ Variable } & $\begin{array}{c}\text { No. of Patients } \\
(\mathbf{\%})\end{array}$ & $\begin{array}{c}\text { Duration of } \\
\text { HAART }\end{array}$ \\
\hline \hline Overall & 101 & $2.9 \pm 1.8$ \\
\hline PI based regimens & $35(34.65)$ & $2.5 \pm 1.5$ \\
\hline NNRTI based regimens & $36(35.64)$ & $2.2 \pm 1.4$ \\
\hline Multiple switching regimens & $30(29.7)$ & $3.8 \pm 2.3$ \\
\hline
\end{tabular}

\section{TREATMENT EFFICACY}

A favorable response to HAART was achieved in 55 (54.5\%), a virological / immunological dissociation occurred in $32(31.7 \%)$, while treatment failure was recorded in 14 (13.9\%) patients (Table 3A). The mean number of drugs needed to achieve a favorable response was $4.8 \pm 2.1$ (range $3-10)$, similar $(P>0.05)$ to the number of drugs taken by those with HAART failure $(5.0 \pm 1.1)$ and a dissociative response $(5.0 \pm 1.5)$.

Gender, age over 40, and HIV transmission risk factor were not associated with HAART failure, nor was clinical AIDS at presentation $(P>0.05)$. Moreover, no specific AIDS-defining condition affected the type of response to HAART $(P=0.33)$, nor did any specific treatment regimen, whether PI- or NNRTI-based.

Ten $(9.9 \%)$ patients died during the follow-up period. The most frequent causes of death were AIDS-related conditions including PML, TB, ADC, and disseminated CMV disease in two cases each, while non-Hodgkin lymphoma and end-stage HCV-related liver disease were the causes of death in one patient each. Of those with a fatal outcome, eight patients $(80 \%)$ experienced HAART failure, and two $(20 \%)$ had a dissociative response to HAART.

The overall estimated survival was eight years (Table 3B). Analysis of factors associated with survival showed that gender, age over 40, HIV disease stage, and HCV coinfection did not affect survival $(P>0.05)$, nor did specific AIDS-defining conditions. Survival was also unaffected by treatment regimen, whether PI- or NNRTI-based $(\mathrm{P}=0.66)$. However, the achievement of undetectable viremia regardless of the degree of immune recovery was a predictor of longer survival (Figs. 1, 2). For patients with a mean viral load of $1.7 \pm 0.23(1.7-1.9)$, the estimated survival was 8.7 years, significantly longer $(P<0.001)$ than in those with a mean viral load of $3.6 \pm 0.82(2.9-5.2)$, whose estimated survival was 4.5 years. Achievement of undetectable viremia during treatment appeared life saving $(\mathrm{OR}=42.5,95 \% \mathrm{CI}$ $7.1-251.9, P=0.000)$, as was as a CD4 cell count of above $200 / \mu \mathrm{L}$ recorded in $57(56.4 \%)$ patients $(\mathrm{OR}=6.4,95 \mathrm{CI}$ 1.2-31.8, $P=0.023)$. The estimated survival in late presenters who achieved a rise in the CD4 cell count to above $200 / \mu \mathrm{L}$ was 8.7 years, as opposed to 5.8 years in those whose $\mathrm{CD}$ cell count did not recover to this level $(P=0.006)$ (Fig. 2). Interestingly, a CD4 count rise to over 400 cells $/ \mu \mathrm{L}$ provided no extra benefit ( $P=0.31, \log$ rank). However, as revealed by multivariate logistic regression, achievement of undetectable viremia was the single independent predictor of survival $(\mathrm{OR}=42.5,95 \% \mathrm{CI} 7.1-251.9, P=0.000)$.

Table 3A. The Outcome of HAART in a Series of 101 Late Presenters

\begin{tabular}{|c|c|}
\hline Variable & No. $(\%)$ \\
\hline \multicolumn{2}{|l|}{ Treatment efficacy } \\
\hline $\mathrm{CD} 4$ count $/ \mu \mathrm{L}($ mean $\pm \mathrm{SD})$ & $265 \pm 198$ \\
\hline $\operatorname{PVL}\left(\log _{10}\right)($ mean \pm SD $)$ & $1.9 \pm 0.6$ \\
\hline Undetectable $\left(\mathrm{pVL} \leq 2.0 \log _{10}\right)$ & $87(86.1)$ \\
\hline Favorable response & $55(54.5)$ \\
\hline Failure & $14(13.9)$ \\
\hline $\begin{array}{l}\text { Virological / immunological dissociation } \\
\text { Adverse events }\end{array}$ & $32(31.7)$ \\
\hline IRD & $11(10.9)$ \\
\hline Severe heptotoxicity & $2(1.9)$ \\
\hline Lipodystophy & $31(30.6)$ \\
\hline Hyperlipidemia & $58(57.4)$ \\
\hline
\end{tabular}

Table 3B. The Estimated Probability of Survival in a Series of 101 Late Presenters (Kaplan Meier)

\begin{tabular}{|c|c|c|}
\hline & Years & $\boldsymbol{P}$ \\
\hline \hline Variable (Yes / No) & $8.7 / 4.5$ & 0.00 \\
Undetectable viremia & $8.7 / 5.8$ & 0.00 \\
CD cell count rose to $>200 / \mu \mathrm{L}$ & & \\
\hline HAART- related survival & 8.1 & 0.66 \\
Only PI-based regimens & 5.3 & \\
Only NNRTI-based regimens & & \\
\hline
\end{tabular}

Immune restoration disease (IRD) developed in 11 (10.9\%) patients, at a mean CD4 cell count of $270.4 \pm 131.0$. Dermatomal herpes zoster was the leading IRD manifestation, appearing in six patients. Other IRD presentations included CMV vitritis, genital herpes, TB, cryptococcal meningitis and a hepatitis flare in an $\mathrm{HCV}$ coinfected patient. The metabolic syndrome occurred frequently during treatment of late presenters. Hyperlipidemia and lipodystrophy were recorded in $58(7.4 \%)$ and $31(30.6 \%)$ patients, respectively. Type 2 diabetes mellitus was recorded in $8(7.9 \%)$. The median time to develop hyperlipidemia and lipodystrophy was 5 and 6 years, respectively. The 9-year probability of developing hyperlipidemia and lipodystrophy was $100 \%$, while it was $30 \%$ for type 2 diabetes mellitus.

\section{DISCUSSION}

According to the results on the efficacy of HAART in our series of late presenters, these severely immunodeficient patients may have a rather good prognosis. Those who initiated HAART during or after recovering from opportunistic infections had a similar life expectancy as 


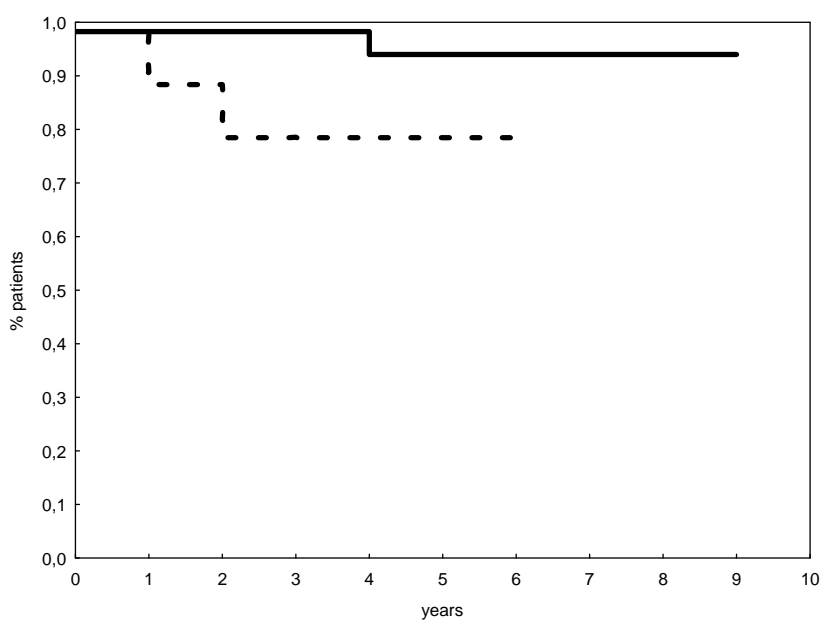

Fig. (1). Estimated probability of survival of late presenters according to achievement of undetectable viremia. $\mathrm{pVL} \leq 2 \log _{10}$ ,pVL $\geq 3 \log _{10}----, P=0.009$.

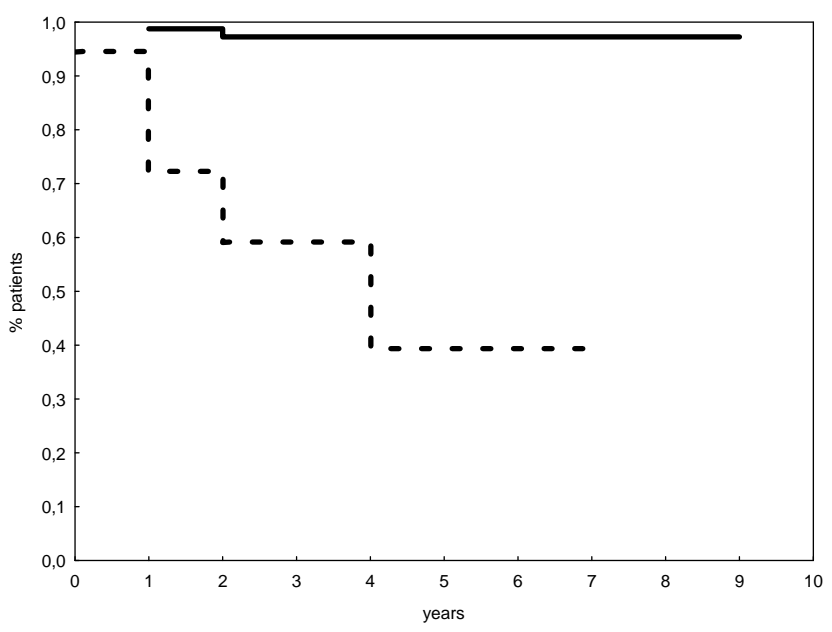

Fig. (2). Estimated probability of survival of late presenters according to achievement of CD4 cell coubt above $200 / \mu \mathrm{L}$ during HAART. Achieved not achieved ----- $P=0.00$.

asymptomatic patients. Specific opportunistic events did not predict the outcome. If patients who commence HAART at a most advanced stage of HIV disease achieve undetectable viremia, they may have a very good prognosis even if the immune system has not recovered to the most desirable CD4 cell counts. Sabin et al. reported a 13\% mortality among late presenters after a follow-up of 2.5 years [7]. If the results in our patient series are analyzed for this period, the mortality rate is quite similar (15.5\%, data not shown). And moreover, since after a mean follow up of $2.9 \pm 1.8$ years the mortality rate dropped to $9.9 \%$, HAART efficacy seems to increase over time. Hocking et al. suggested that increased survival of late presenters with AIDS on HAART may indicate a positive response of drug naive patients to antiretroviral therapies [6].

Using the life expectancy model for the prognosis of HIV/AIDS in the HAART era, the ART Cohort Collaboration study reported a $8.5 \%$ mortality rate for patients who initiated HAART at a CD4 cell count below
$50 / \mu \mathrm{L}[5,6]$. Even in the high income countries analyzed by the ART Cohort Collaboration study, late presentation had emerged as an issue of concern, since nearly one half of patients started HAART either with a CD4 cell count of less than 200 cells/ $\mu \mathrm{L}$ or with a diagnosis of AIDS. HIV management guidelines advocate early diagnosis and timely treatment. Such a strategy is essential to prevent clinical progression as well as the spread of the infection, in line with public health principles of wide screening and treatment shown to be effective in the control of many infectious diseases. The Centres for Disease Control and Prevention estimate that more than one half of new HIV infections are spread by HIV-positive individuals who are unaware of their infection, and thus support expanded, voluntary and costeffective screening in healthcare settings, on the basis of optout strategy [21]. Wide voluntary screening in healthcare settings should be particularly advocated in Serbia, given that the HIV testing rate in Serbia is among the lowest in Europe.

Profound immunodeficiency on HAART initiation is associated with a risk of IRD. We, and others, have previously shown that a CD4 count below $100 / \mu \mathrm{L}$ at HAART initiation is a major risk factor for the development of IRD during HAART $[22,23]$. The overall prevalence of IRD among late presenters was $10.9 \%$, similar to our earlier data in the whole Serbian cohort of HIV/AIDS patients $(16.7 \%)$ [22]. IRD strongly affects the patients' quality of life, since these episodes often require hospital admissions. Hyperlipidemia and lipodystrophy also emerged as important issues among late presenters on HAART, with an increased incidence as compared to the whole cohort [24].

Taken together, the presented results on HAART efficacy in late presenters indicate that reaching and maintaining undetectable viremia is a prerequisite for good prognosis for late presenters on HAART, regardless of the level of immune recovery.

\section{ACKNOWLEDGEMENT}

The work has been supported by grant M145002 from the Ministry of Science and Technological Development of Serbia.

\section{REFERENCES}

[1] Jacobson MA, French MA. Altered natural history of AIDS-related opportunistic infections in the era of potent combination antiretroviral therapy. AIDS 1998; 12 (suppl. A): S157-S163.

[2] Gea-Banacloche JC, Lane $\mathrm{CH}$. Immune reconstitution in HIV infection. AIDS 1999; 13 (suppl A): S25-S38.

[3] Carcelain G, Li T, Autran B. Immune reconstitution under highly active antiretroviral therapy (HAART). AIDS Rev 1999; 1: 51-6.

[4] Jevtović Dj, Salemović D, Ranin J, Pešić I, Žerjav S, DjurkovićDjaković O. Long-term survival of HIV-infected patients treated with highly active antiretroviral therapy in Serbia and Montenegro. HIV Med 2007; 8: 75-9.

[5] The Antiretroviral Therapy Cohort Collaboration. Life expectance of individuals on combination antiretro-viral therapy in highincome countries: a collaborative analysis of 14 cohort studies. Lancet 2008; 372: 393-9.

[6] The Antiretroviral Therapy (ART) Cohort Collaboration. Prognosis of HIV-1 infected patients up to 5 years after initiation of HAART: collaborative analysis of prospective studies. AIDS 2007; 21: 118597.

[7] Sabin CA, Smith CJ, Gumley H, et al. Late presenters in the era of highly active antiretroviral therapy: uptake of and response to antiretroviral therapy. AIDS 2004; 18: 2145-51. 
[8] Sabin CA, Smith CJ, Youle M, et al. Death in the era of HAART: contribution of late presentation, treatment exposure, resistance and abnormal laboratory markers. AIDS 2006; 20: 67-71.

[9] Hocking JS, Rodger AJ, Rhodes DG, Crofts N. Late presentation of HIV infection associated with prolonged survival following AIDS diagnosis: characteristics of individuals. Int J STD AIDS 2000; 11: 503-8.

[10] Girardi E, Aloisi MS, Arici C, et al. Delayed presentation and late testing for HIV: demographic and behavioral risk factors in multicenter study in Italy. J Acquir Immune Defic Syndr 2004; 36: 951-9.

[11] Nunez M, Asencio R, Valencia ME, Leal M, Gonzales-Lahoz J, Soriano V. Rate, causes, and clinical implications of presenting with low CD4 cell counts in the era of highly active antiretroviral therapy. AIDS Res Hum Retrovir 2003; 19: 363-8.

[12] Wong KH, Lee SS, Low KH, Wan WY. Temporal trends and factors associated with late diagnosis in Hong Kong, a low HIV prevalent locality. AIDS Patient Care STDS 2003: 17: 461-9.

[13] Jesus C, Paz S, de la Fuente L, Noguer I, Luis G, Parras F. Late diagnosis of HIV infection in the era of highly active antiretroviral therapy: consequences for AIDS incidence. AIDS 2002; 16: 1945-51.

[14] Mocroft A, Decereux H, Kinloch-de-Loes S, et al. Immunological, virological and clinical response to highly active antiretroviral therapy regimens in a complete clinical population. Royal Free Centre for HIV Medicine. AIDS 2000, 14: 1545-52.

[15] Kilaru KR, Kimar A, Sippy N, Carter AO, Roach TC. Immunological and virological response to highly active antiretroviral therapy in a non-clinical trial setting in a developing Caribbean country. HIV Med 2006; 7: 99-104.

[16] Rizzardi GP, Tambussi G, Bart PA, Chapuis AG, Lazzarin A, Pantaleo G. Virological and immunological response to HAART in asymptomatic therapy-naive $\mathrm{HIV}-1$ infected subjects according to CD4 cell count. AIDS 2000; 14: 2257-63.

[17] CDC. 1993 revised classification system for HIV infection and expanded surveillance case definition for AIDS among adolescents and adults. MMWR 1992; 41[OR-17]: 1-19.

[18] Pakker NG, Kroon ED, Roos MT, et al. Immune restoration does not invariably occur following long-term HIV-1 suppression during antiretroviral therapy. INCAS Study Group. AIDS 1999; 13: 20312.

[19] Jevtović Dj, Salemović D, Ranin J, Pešić I, Žerjav S, DjurkovićDjaković O. The dissociation between virological and immunological responses to HAART. Biomed Pharmacother 2005; 59: 44651.

[20] Durant J, Clebenbergh P, Halfon P, et al. Drug-resistance genotyping in HIV-1 therapy: the VIRADEPT randomised controlled trial. Lancet 1999; 353: 2195-9.

[21] Advancing HIV prevention: new strategies for a changing epidemic - United States, 2003. MMWR 2003; 52: 329-32.

[22] Jevtović Dj, Salemović D, Ranin J, Pesić I, Žerjav S, DjurkovićDjaković O. The incidence and risks for immune restoration disease in HAART-treated HIV patients treated with highly active antiretroviral therapy. HIV Med 2005; 6: 140-3.

[23] Murdoch DM, Venter WDF, Van Rie A, Fildman C. Immune reconstitution inflammatory syn-drome (IRIS): review of common infectious manifestations and treatment options. AIDS Res Ther 2007; 4: 1-10

[24] Jevtović Dj, Dragović G, Salemović D, Ranin J, Djurković Djaković O. The metabolic syndrome, an epidemic among HIV infected patients on HAART. Biomed Pharmacother 2009; 63: 337 42.

(C) Jevtović et al.; Licensee Bentham Open.

This is an open access article licensed under the terms of the Creative Commons Attribution Non-Commercial License (http: //creativecommons.org/licenses/by-nc/ 3.0/) which permits unrestricted, non-commercial use, distribution and reproduction in any medium, provided the work is properly cited. 